\title{
Prognostic scores in cirrhotic patients admitted to a gastroenterology intensive care unit
}

\author{
Paulo Freire, José M. Romãozinho, Pedro Amaro, Manuela Ferreira and Carlos Sofia \\ Department of Gastroenterology. Coimbra University Hospital. Coimbra, Portugal
}

\begin{abstract}
Background: prognostic scores have been validated in cirrhotic patients admitted to general Intensive Care Units. No assessment of these scores was performed in cirrhotics admitted to specialized Gastroenterology Intensive Care Units (GICUs).

Aim: to assess the prognostic accuracy of Acute Physiology and Chronic Health Evaluation (APACHE) II, Simplified Acute Physiology Score (SAPS) II, Sequential Organ Failure Assessment (SOFA), Model for End-stage Liver Disease (MELD) and ChildPugh-Turcotte (CPT) in predicting GICU mortality in cirrhotic patients.

Methods: the study involved 124 consecutive cirrhotic admissions to a GICU. Clinical data, prognostic scores and mortality were recorded. Discrimination was evaluated with area under receiver operating characteristic curves (AUC). Calibration was assessed with Hosmer-Lemeshow goodness-of-fit test.

Results: GICU mortality was 9.7\%. Mean APACHE II, SAPS II, SOFA, MELD and CPT scores for survivors (13.6, 25.4, 3.5, 18.0 and 8.6, respectively) were found to be significantly lower than those of non-survivors $(22.0,47.5,10.1,30.7$ and 12.5, respectively) $(p<0.001)$. All the prognostic systems showed good discrimination, with $\mathrm{AUC}=0.860,0.911,0.868,0.897$ and 0.914 for APACHE II, SAPS II, SOFA, MELD and CPT, respectively. Similarly, APACHE II, SAPS II, SOFA, MELD and CPT scores achieved good calibration, with $\mathrm{p}=0.146,0.120,0.686$, 0.267 and 0.120 , respectively. The overall correctness of prediction was $81.9 \%, 86.1 \%, 93.3 \%, 90.7 \%$ and $87.7 \%$ for the APACHE II, SAPS II, SOFA, MELD and CPT scores, respectively.

Conclusions: in cirrhotics admitted to a GICU, all the tested scores have good prognostic accuracy, with SOFA and MELD showing the greatest overall correctness of prediction.
\end{abstract}

Key words: Gastroenterology Intensive Care Unit. Cirrhosis. APACHE II. SAPS II. SOFA. MELD. Child-Pugh-Turcotte. Prognostic scores.

\section{Received: 04-10-10.}

Accepted: 02-12-10.

Correspondence: Paulo André Vinagreiro Freire. Department of Gastroenterology. Coimbra University Hospital. Av. Bissaya Barreto - Praceta Prof. Mota Pinto. 3000-075 Coimbra, Portugal

e-mail: pauloavfreire@gmail.com
Freire Paulo, Romãozinho José M., Amaro Pedro, Ferreira Manuela, Sofia Carlos. Prognostic scores in cirrhotic patients admitted to a gastroenterology intensive care unit. Rev Esp Enferm Dig 2011; 103: 177-183.

\section{INTRODUCTION}

Prognostic models are used for comparison and quality assessment of different Intensive Care Units (ICUs) and within the same ICU over time, for audit and clinical research, for evaluating therapeutic effectiveness and for guiding discussions between clinicians and families (1-6).

The incidence of liver cirrhosis is increasing exponentially and cirrhotic patients admitted to ICUs have high mortality rates and a high rate of consumption of resources (7-18). Furthermore, a significant percentage (36.7\%) of the total cost of ICU care for cirrhotic patients is spent on those who do not survive $(11,16,19)$. Additionally, it must be borne in mind that ICUs are a very limited health resource $(10,11,18)$. Thus, predictive models could be very important decision-making tools in this setting.

Two types of prognostic scores can be used in cirrhotics admitted to ICUs: general prognostic models and disease-specific models. The general prognostic models include two main categories: firstly, those evaluating severity of illness, namely the Acute Physiology and Chronic Health Evaluation (APACHE) II (20) and Simplified Acute Physiology Score (SAPS) II (21); secondly, models quantifying organ dysfunction and failure, of which the most widely-used is the Sequential Organ Failure Assessment (SOFA) $(22,23)$. These scores have been validated to predict survival in general ICU populations (with few cirrhotics included in the original model devel- 
opment) using data collected within the first $24 \mathrm{~h}$ in ICU (1,24-29). Specific scoring systems for liver disease include Child-Pugh-Turcotte (CPT) (30,31) and the Model for End-stage Liver Disease (MELD) (32). CPT was originally developed and later modified to predict mortality in patients undergoing surgical treatment for variceal bleeding and MELD was developed to predict mortality in patients undergoing trans-jugular intrahepatic portosystemic shunt and is currently used to predict shortterm survival in cirrhotics and to prioritize recipients of transplants (30-33). Its usefulness and excellent performance in these situations led to research into its application in other areas, including risk assessment of cirrhotic patients admitted to ICUs (29).

In a review article on studies that assessed the performance of these scores in predicting the mortality of cirrhotics admitted to ICUs, the authors concluded that usually general ICU-models have better discrimination than liver specific models and that SOFA has the best predictive ability among the general scores (29). These results are somewhat unsurprising if we bear in mind that the prognosis of acutely ill patients with cirrhosis is influenced not only by the severity of hepatic insufficiency but mainly by the dysfunction of other organ systems and that cirrhotics admitted to ICUs often have extrahepatic organ dysfunction and die of multiple organ failure (1214,34-39).

Moreover, it is known that patients with different disease status and/or indications for ICU admission have widely different outcomes (40). In fact, cirrhotics admitted with septic shock have a worse outcome than those admitted only for airway protection after gastrointestinal bleeding (35). This means that the above conclusions are only valid for cirrhotics admitted to general ICUs and cannot be extrapolated to other sub-populations, namely to cirrhotics admitted to specialized Gastroenterology Intensive Care Units (GICUs). To our knowledge, no reports are available on validation of these prognostic models in patients with liver cirrhosis admitted to GICUs.

Thus, the aim of this study was to assess the prognostic accuracy of APACHE II, SAPS II, SOFA, MELD and CPT scores to predict GICU mortality in patients with liver cirrhosis.

\section{PATIENTS AND METHODS}

\section{Patient information}

This retrospective study included 124 consecutive hospitalisations of 114 patients with liver cirrhosis admitted to a 4-bed specialized GICU in a 1200-bed Portuguese university hospital between February 2005 and October 2006. In this unit are admitted patients with hepatic and/or gastrointestinal severe medical diseases. Surgical patients, including post-transplant, are not admitted in this GICU, since there are specific units in our hospital for these patients. Patients admitted for less than one day were excluded from the study. For the purposes of this study, each admission was considered a separate patient, as previously reported (41).

The diagnosis of cirrhosis was based on liver biopsy ( $\mathrm{n}$ $=32 ; 25.8 \%)$ or clinical evaluation $(\mathrm{n}=92 ; 74.2 \%)$. The clinical diagnosis of cirrhosis was made by a history of portal hypertension excluding other etiology, evidence of esophageal varices confirmed by endoscopy, impaired liver function tests, impaired clotting parameters and ultrasonographic or computer tomographic criteria (41-44).

The following data was collected: demographics, etiology of cirrhosis, indication for admission, APACHE II, SAPS II, SOFA, MELD and CPT scores and survival on GICU discharge. The scores were computed for each patient using data collected within the first $24 \mathrm{~h}$ in GICU, selecting the worst (most abnormal) value for each variable during this period, as recommended (29). All the above scoring systems were calculated as published (2023,30-32).

The main study outcome was GICU mortality.

\section{Ethical approval}

The local Ethics Committee approved this research.

\section{Statistical analysis}

Continuous variables were summarized using means and standard error and normal distribution was assessed by the Kolmogorov-Smirnov test. The Student s $t$-test was employed to compare the means of normally distributed data; otherwise, the Mann-Whitney $U$-test was employed. Categorical variables were expressed as frequency and percentage and the corresponding contingency tables were analyzed with $\chi^{2}$ test. A $p$ value of less than 0.05 was considered statistically significant.

Model performance is usually statistically evaluated by measuring calibration and discriminative ability $(24,45)$. Calibration measures the degree of correspondence between predicted and observed mortality over the entire range of risks, whilst discrimination gauges the model's ability to differentiate between the patients who died and those who survived. Calibration was described by goodness-of-fit testing using the Hosmer-Lemeshow test and considering $p$ values $\geq 0.1$ as good calibration (46). Discrimination was evaluated by using the area under receiver operation characteristic (ROC) curve (AUC), for which values above 0.8 were considered good (clinically useful) (47).

The Youden index (sensitivity + specificity -1) (48) was used to select the best cut-off points, at which positive predictive value, negative predictive value, sensitivity, specificity, overall correctness of prediction and odds ratio for GICU mortality were calculated. 
Table I. Indication for GICU admission

\begin{tabular}{lll}
\hline Primary cause of GICU admission & $n$ & (\%) \\
\hline Variceal bleeding & 81 & $(65.3)$ \\
Bleeding peptic ulcer & 20 & $(16.1)$ \\
Hepatic encephalopathy & 9 & $(7.3)$ \\
Acute pancreatitis & 4 & $(3.2)$ \\
Miscellaneous/other causes & 10 & $(8.1)$ \\
\hline
\end{tabular}

GICU: Gastroenterology Intensive Care Unit.

The data was analyzed using the Statistical Package for Social Sciences-SPSS (SPSS Inc., Chicago, Illinois, USA) computer software for Windows (version 17.0).

\section{RESULTS}

A total of 114 patients with 124 admissions to GICU were studied. Of the 124 patient admissions, 89 (71.8\%) were men and $35(28.2 \%)$ were women. The mean age was $57.7 \pm 14.1$ years (range, $18-89$ years) and the average length of stay in GICU was $5.5 \pm 5.1$ (range 2-35).

Indication for GICU admission was mostly due to upper gastrointestinal bleeding $(81.4 \%, \mathrm{n}=101$; Table I), including $81(65.3 \%)$ patients with variceal bleeding and $20(16.1 \%)$ with bleeding peptic ulcers. Of the 81 patients admitted with variceal bleeding, 69 patients had esophageal variceal bleeding and 12 gastric variceal bleeding. The primary treatment for esophageal variceal bleeding was elastic band ligation, sclerosis (with absolute alcohol) and Sengstaken-Blackmore balloon tamponade in $30(43.5 \%), 21(30.4 \%)$ and $18(26.1 \%)$ patients, respectively. In the 12 patients admitted with gastric variceal bleeding the primary treatment was Histoacryl $^{\oplus}+$ Lipiodol in $11(91.7 \%)$ patients and Sengstaken-Blackmore balloon tamponade in $1(8.3 \%)$ patient.

At admission $23(18.5 \%), 4(3.2 \%), 5(4.0 \%)$ and 1 $(0.8 \%)$ patients met criteria of systemic inflammatory response (SIRS), sepsis, severe sepsis and multiple organ dysfunction, respectively.

Alcohol was the most frequent etiology of cirrhosis $(80.6 \%, \mathrm{n}=100)$. The remaining etiologies of the liver disease are shown in table II.

Acute renal failure or acute-on-chronic renal failure was found in $27(21.8 \%)$ patients, requiring dialysis in 5 patients $(4.0 \%)$. Nineteen patients $(15.3 \%)$ required endotracheal intubation, including $13(10.5 \%)$ with mechanical ventilation.

The mean \pm standard deviation (and range) of the APACHE II, SAPS II, SOFA, MELD and CPT scores were $14.4 \pm 6.8$ (2-44), $27.5 \pm 12.8$ (6-72), 4,1 $\pm 3,3(0-16), 19.3 \pm$ 8.7 (2-55) and $9.0 \pm 2.5$ (5-14), respectively. Overall GICU mortality was $9.7 \%(n=12)$. The non-survivors had significantly higher average values of the tested scores (Table III).
Table II. Etiologies of liver cirrhosis

\begin{tabular}{lll}
\hline Etiology of liver cirrhosis & $n$ & (\%) \\
\hline Alcohol & 100 & $(80.7)$ \\
Hepatitis C virus & 7 & $(5.7)$ \\
Hepatitis B virus & 6 & $(4.8)$ \\
Primary biliary cirrhosis & 5 & $(4.0)$ \\
Autoimmune & 3 & $(2.4)$ \\
Cryptogenic & 3 & $(2.4)$ \\
\hline
\end{tabular}

All the prognostic systems achieved AUC $>0.80$, thus revealing good discrimination. The SAPS II and CPT scores, with AUC $=0.911$ and 0.914 , respectively, showed the best discriminative accuracy. ROC curves and the corresponding AUC are illustrated in figure 1.

Regarding calibration, goodness-of-fit, as measured by the Lemeshow-Hosmer test, displayed $p>0.1$ for all the scores, confirming that predicted mortality was similar to observed mortality (i.e. good calibration) (Table IV). The SOFA and MELD scores, with $p=0.686$ and 0.267 , respectively, achieved the best calibration (Table IV).

The best Youden index and the resultant cut-off points are shown in table IV. Table V reports positive predictive value, negative predictive value, sensitivity, specificity and overall correctness of prediction at the cut-off points obtained. Finally, the GICU mortality rates above and below the cut-off points and the corresponding odds ratio for GICU mortality are detailed in table VI.

\section{DISCUSSION}

Accurate prognostic indicators for patient survival in ICUs can be used by clinicians to frame discussions with families and possibly to guide limitation of further intervention in extremely ill patients (3-6). This is particularly relevant in cirrhotic patients, owing to the exponential increase in the incidence of this condition and the limited ICU resources (7-19).

Table III. Average values of APACHE II, SAPS II, SOFA, MELD and CPT scores according to survival

\begin{tabular}{llll}
\hline Scoring system & Survivors & Non-survivors & p value \\
\hline APACHE II & $13.6 \pm 6.4$ & $22.0 \pm 6.1$ & $<0.001$ \\
SAPS II & $25.4 \pm 10.7$ & $47.5 \pm 13.6$ & $<0.001$ \\
SOFA & $3.5 \pm 2.7$ & $10.1 \pm 3.0$ & $<0.001$ \\
MELD & $18.0 \pm 7.6$ & $30.7 \pm 10.1$ & $<0.001$ \\
CPT & $8.6 \pm 2.3$ & $12.5 \pm 1.7$ & $<0.001$
\end{tabular}

APACHE: Acute Physiology and Chronic Health Evaluation; SAPS: Simplified Acute Physiology Score; SOFA: Sequential Organ Failure Assessment; MELD: Model for End-stage Liver Disease; CPT: Child- Pugh-Turcotte. 


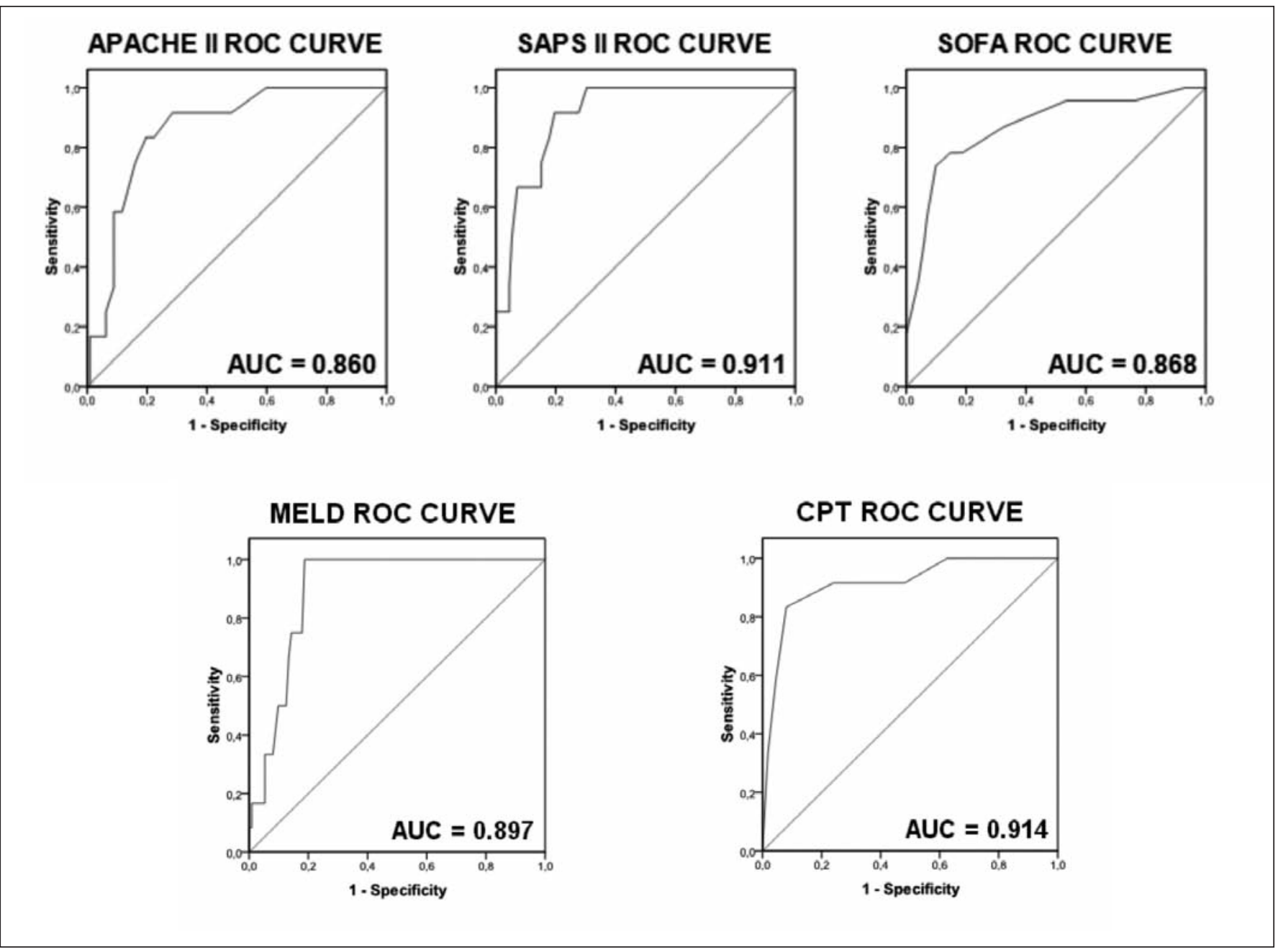

Fig. 1. Receiver operation characteristic (ROC) curves and corresponding area under the curve (AUC) for Acute Physiology and Chronic Health Evaluation (APACHE) II, Simplified Acute Physiology Score (SAPS) II, Sequential Organ Failure Assessment (SOFA), Model for End-stage Liver Disease (MELD) and Child-Pugh-Turcotte (CPT) in 124 consecutive cirrhotic admissions to a Gastroenterology Intensive Care Unit.

Our study assessed, to our knowledge for the first time, the accuracy of prognostic scores in cirrhotic patients admitted to a GICU. In our series, all the tested

Table IV. Calibration, Youden Index and cut-off values of the scoring systems

\begin{tabular}{llll}
\hline Scoring system & $\begin{array}{l}\text { Calibration } \\
\text { goodness-of-fit }\left(\chi^{2}\right)\end{array}$ & Youden index & Cut-off point \\
\hline APACHE II & 0.146 & 0.80 & 18 \\
SAPS II & 0.120 & 0.80 & 34 \\
SOFA & 0.686 & 0.90 & 7 \\
MELD & 0.267 & 0.81 & 23 \\
CPT & 0.120 & 0.92 & 12
\end{tabular}

APACHE: Acute Physiology and Chronic Health Evaluation; SAPS: Simplified Acute Physiology Score; SOFA: Sequential Organ Failure Assessment; MELD: Model for End-stage Liver Disease; CPT: Child- Pugh-Turcotte. scores displayed good discrimination and calibration. This means that these scores are able to classify patients correctly as survivors or non-survivors and that modelpredicted mortality was similar to observed mortality. Regarding discrimination, the best performance was achieved by SAPS II and CPT scores with AUC $=0.911$ and 0.914, respectively (Fig. 1). Concerning calibration, the best results were obtained by SOFA and MELD scores with $p=0.686$ and 0.267 , respectively (Table IV).

The excellent performance of all the tested scores in our study is also reflected in the high sensitivity, specificity and overall correctness of prediction (Table V). The SOFA and MELD scores, with an overall correctness of prediction of 93.3 and $90.7 \%$ respectively, showed the best predictive ability (Table V). These findings support the importance of evaluating the performance of a predictive model not only in terms of its discriminative capacity but also its calibration. If, as in some studies $(13,17,36,38)$, we had based the evaluation on discriminative ability alone, 
Table V. Performance in prediction of GICU mortality

\begin{tabular}{lccccc}
\hline Scoring system & PPV (\%) & NPV (\%) & Sensitivity (\%) & Specificity (\%) & Overall correctness of prediction (\%) \\
\hline APACHE II & 31.3 & 97.8 & 83.3 & 80.4 & 81.9 \\
SAPS II & 33.3 & 91.7 & 91.7 & 80.4 & 86.1 \\
SOFA & 44.4 & 100 & 100 & 86.6 & 93.3 \\
MELD & 36.4 & 100 & 100 & 81.3 & 90.7 \\
CPT & 52.6 & 98.1 & 83.3 & 92.0 & 87.7
\end{tabular}

GICU: Gastroenterology Intensive Care Unit; PPV: Positive Predictive Value; NPV: Negative Predictive Value; APACHE: Acute Physiology and Chronic Health Evaluation; SAPS: Simplified Acute Physiology Score; SOFA: Sequential Organ Failure Assessment; MELD: Model for End-stage Liver Disease; CPT: Child- Pugh-Turcotte.

we would say that the SAPS II and CPT scores had the best performance. Nevertheless, we found that the best calibration results were obtained by the SOFA and MELD scores. The importance of calibration is reflected in the fact that the best overall correctness of prediction was achieved by the SOFA and MELD scores. Thus, our data underline that we must look carefully to the results of studies in which prognostic performance is evaluated only in terms of discriminative ability.

Originally the tested scores were not developed to assess the prognosis of cirrhotic patients admitted to ICU. The general scores were developed and calibrated to predict outcome in general ICUs, with few cirrhotics included in the original model development (20-23). The liverspecific scores were developed to assess liver function and prognosis in specific clinical situations, namely prediction of mortality in patients undergoing surgical or intravascular treatment of portal hypertension (30-32). Nonetheless, both general and liver-specific scores have been later evaluated and validated as prognostic models in cirrhotics admitted to general ICUs. However, it is a well known fact that mortality prediction model performance usually deteriorates when models are applied to different population samples, i.e. less sick patients. Indeed, although APACHE II works well for severely ill cirrhotic patients admitted to ICUs $(13,17,29,36-38)$, this is not the case when its predictive value is assessed in a population of cirrhotic patients with mortality rate of only $11.5 \%$ (41). Thus, our results are somewhat surprising, because the mortality rate in our sample was only $9.7 \%$ and nevertheless the scores showed an excellent performance. However, as expected in a population with a relatively low mortality rate, this excellent performance is reflected in high negative predictive values but relatively low positive predictive values (Table V). Nonetheless, these relatively low positive predictive values are strong enough to justify a significantly higher mortality in patients with score values above the cut-off points (Table VI).

Cholongitas et al., in a review article on prognostic scores in cirrhotic patients admitted to ICUs, concluded that overall the general scores provide better a prognosis prediction than the liver specific scores and also that SOFA reveals the best predictive ability amongst the general scores (29). These results were somewhat expected if we take into account the characteristics of the cirrhotic patients admitted to ICUs and the structure of the different scores. Regarding the characteristics of the cirrhotics admitted to ICUs, these patients often have extrahepatic organ dysfunction and die of multiple organ failure (1214,34-39). Concerning the structure of the prognostic models, the general scores include a global assessment of the patient's clinical condition, evaluating parameters related to the various organ systems (20-23). On the other hand, liver-specific scores evaluate the hepatic function

Table VI. Cut-off points: mortality rate and Odds Ratio for GICU mortality

\begin{tabular}{llcll}
\hline Scoring system & & Mortality rate (\%) & $p$ value & Odds ratio for GICU mortality (95\% Cl) \\
\hline APACHE II & $\geq 18$ & 31.3 & $<0.001$ & $20.46(4.18-100.12)$ \\
& $<18$ & 2.2 & & \\
SAPS II & $\geq 34$ & 33.3 & $<0.001$ & $45.00(5.51-367.30)$ \\
& $<34$ & 1.1 & $<0.001$ & \\
SOFA & $\geq 7$ & 44.4 & $<$ Infinite \\
MELD & $\geq 7$ & 0 & $<0.001$ & Infinite \\
CPT & $\geq 23$ & 36.4 & $<0.001$ & $57.22(10.84-302.17)$
\end{tabular}

GICU: Gastroenterology Intensive Care Unit; APACHE: Acute Physiology and Chronic Health Evaluation; SAPS: Simplified Acute Physiology Score; SOFA: Sequential Organ Failure Assessment; MELD: Model for End-stage Liver Disease; CPT: Child- Pugh-Turcotte; CI: Confidence interval. 
exclusively, as in the CTP classification, or supplement this evaluation with an assessment of the renal function, as the MELD score does (30-33). In our study, beside SOFA being the score with higher diagnostic accuracy, we found that the liver specific scores MELD and CPT showed better predictive ability than the APACHE II and SAPS II general scores. These results may suggest that in GICUs the relative importance of the severity of hepatic insufficiency and extrahepatic organ dysfunction is different from general ICUs. In other words, it can be said that the prognostic importance of the degree of liver failure appears to be higher in GICUs than in general ICUs.

In addition to predictive accuracy, for any predictive model to be clinically useful it must also show reproducibility and be easy to use at the bedside $(49,50)$. The APACHE II and SAPS II scores are calculated with objective variables included in the standard monitoring of critically ill patients, but their use in daily routines has been impeded by the need to process data by computer $(17,18,20,21,40,44)$. The MELD score takes objective parameters into consideration and requires little data, but bedside use is also limited by the need for computerized calculations $(18,32,33,44)$. CPT is a simple score that can be easy and quickly determined without the need for computer calculation, but its reproducibility is compromised by the subjectivity of some of its variables $(30-32,44)$. The SOFA is the most attractive score for use in daily clinical practice, because the variables it requires are derived from standard monitoring of critically ill patients and bedside calculation takes only 3 minutes $(22,23,29,51)$.

Therefore, SOFA is a user-friendly score that combines high reproducibility with excellent predictive ability $(22,23,29,51)$. However, there are some limitations of the SOFA that should be addressed. Firstly and most importantly with regard to the population under consideration, the liver function is evaluated with serum bilirubin although this has a limited ability to reflect the full spectrum of liver dysfunction in critical illness and cannot differentiate between acute liver dysfunctions and the effects of pre-existing chronic disease (52). Secondly, diagnosis, age and co-morbid conditions are ignored. Finally, the neurological variable assessed is the Glasgow Coma Scale, but its clinical evaluation is subjective and it is affected by sedative and analgesic drugs frequently administered to critically ill patients.

Apart from the limitations and/or drawbacks of each score and although the performance of all the tested scores was excellent in our study, they share some structural limitations in the prognostic assessment of cirrhotic patients. Transfused blood units, for example, are not measured by any of the tested scores and presumably this is an important prognostic variable in a population in which the major reason for admission is upper gastrointestinal bleeding. Therefore, in future, it would be interesting to develop studies to identify independent risk factors in cirrhotic patients admitted to a GICU and, on this basis, to develop a specific score in this setting.
Notwithstanding the encouraging results, this study has some limitations. Firstly, it is a retrospective study. Secondly, the work was conducted in a single academic referral hospital; consequently, the results may not be directly extrapolated to other patient populations. Finally, patients with a GICU stay $<24$ hours were excluded, resulting in a mortality rate of only $9.7 \%$. It could be argued that the rationale for excluding these patients undermines our study, as the most likely scenario is that these patients have died. However, this problem is shared by all the other works in this area, as the tested scores, by definition, must be calculated with the worst value for each variable obtained during the first $24 \mathrm{~h}$ of admission (29).

Although prognostic scores are important in the decision-making process, it must be borne in mind that no model can predict the outcome for a patient with $100 \%$ accuracy (53-55). Thus, prognostic models should be used as tools to supplement, rather than substitute, clinical assessment.

In conclusion, our data showed that the general prognostic scores APACHE II, SAPS II and SOFA and the liverspecific scores MELD and CPT display good discrimination and calibration for predicting mortality in cirrhotic patients admitted in GICUs and, therefore, are clinically useful in this context. Furthermore, our results indicate that from amongst these scores, SOFA and MELD have the greatest overall correctness of prediction. Nevertheless, a validation of our results is required in other GICUs, preferably in large prospective multicentre studies. The clinical evaluation of patients cannot be replaced by prognostic scores. However, we believe that these prognostic models may represent an important objective tool in the assessment of patient prognosis and, hence, may be a valuable complement to clinical evaluation in the decision-making process.

\section{REFERENCES}

1. Becker RB, Zimmerman JE. ICU scoring systems allow prediction of patient outcomes and comparison of ICU performance. Crit Care Clin 1996;12:503-14.

2. Sherck JP, Shatney CH. ICU scoring systems do not allow prediction of patient outcomes or comparison of ICU performance. Crit Care Clin 1996;12:515-23.

3. Pronovost PJ, Nolan T, Zeger S, Miller M, Rubin H. How can clinicians measure safety and quality in acute care? Lancet 2004;363:1061-7.

4. Gropper MA. Evidence-based management of critically ill patients: analysis and implementation. Anesth Analg 2004;99:566-72.

5. Friedman Y. Ethical issues in the critically ill patient. Curr Opin Crit Care 2001;7:475-9.

6. Azoulay E, Sprung CL. Family-physician interactions in the intensive care unit. Crit Care Med 2004;32:2323-8.

7. Austin MJ, Shawcross DL. Outcome of patients with cirrhosis admitted to intensive care. Curr Opin Crit Care 2008;14:202-7.

8. Cullen DJ, Ferrara LC, Briggs BA, Walker PF, Gilbert J. Survival, hospitalization charges and follow-up results in critically ill patients. N Engl J Med 1976;294:982-7.

9. Zook CJ, Moore FD. High-cost users of medical care. N Engl J Med 1980;302:996-1002.

10. Thibault GE, Mulley AG, Barnett GO, Goldstein RL, Reder VA, Sherman EL, et al. Medical intensive care: indications, interventions, and outcomes. N Engl J Med 1980;302:938-42. 
11. Detsky AS, Stricker SC, Mulley AG, Thibault GE. Prognosis, survi$\mathrm{val}$, and the expenditure of hospital resources for patients in an intensive-care unit. N Engl J Med 1981;305:667-72.

12. Shellman RG, Fulkerson WJ, DeLong E, Piantadosi CA. Prognosis of patients with cirrhosis and chronic liver disease admitted to the medical intensive care unit. Crit Care Med 1988;16:671-8.

13. Zimmerman JE, Wagner DP, Seneff MG, Becker RB, Sun X, Knaus WA. Intensive care unit admissions with cirrhosis: risk-stratifying patient groups and predicting individual survival. Hepatology 1996; 23:1393-401.

14. Singh N, Gayowski T, Wagener MM, Marino IR. Outcome of patients with cirrhosis requiring intensive care unit support: prospective assessment of predictors of mortality. J Gastroenterol 1998;33:73-9.

15. Kress JP, Rubin A, Pohlman AS, Hall JB. Outcomes of critically ill patients denied consideration for liver transplantation. Am J Respir Crit Care Med 2000;162:418-23.

16. Wong LL, McFall P, Wong LM. The cost of dying of end-stage liver disease. Arch Intern Med 1997;157:1429-32.

17. Aggarwal A, Ong JP, Younossi ZM, Nelson DR, Hoffman-Hogg L, Arroliga AC. Predictors of mortality and resource utilization in cirrhotic patients admitted to the medical ICU. Chest 2001;119:1489-97.

18. Cholongitas E, Senzolo M, Patch D, Kwong K, Nikolopoulou V, Leandro G, et al. Risk factors, sequential organ failure assessment and model for end-stage liver disease scores for predicting short term mortality in cirrhotic patients admitted to intensive care unit. Aliment Pharmacol Ther 2006;23:883-93.

19. Gildea TR, Cook WC, Nelson DR, Aggarwal A, Carey W, Younossi $\mathrm{ZM}$, et al. Predictors of long-term mortality in patients with cirrhosis of the liver admitted to a medical ICU. Chest 2004;126:1598-603.

20. Knaus WA, Draper EA, Wagner DP, Zimmerman JE. APACHE II: a severity of disease classification system. Crit Care Med 1985;13:818-29.

21. Le Gall JR, Lemeshow S, Saulnier F. A new Simplified Acute Physiology Score (SAPS II) based on a European/North American multicenter study. JAMA 1993;270:2957-63.

22. Vincent JL, Ferreira F, Moreno R. Scoring systems for assessing organ dysfunction and survival. Crit Care Clin 2000;16:353-66.

23. Vincent JL, Moreno R, Takala J, Willatts S, De Mendonça A, Bruining H, et al. The SOFA (Sepsis-related Organ Failure Assessment) score to describe organ dysfunction/failure. On behalf of the Working Group on Sepsis-Related Problems of the European Society of Intensive Care Medicine. Intensive Care Med 1996;22:707-10.

24. Strand K, Flaatten H. Severity scoring in the ICU: a review. Acta Anaesthesiol Scand 2008;52:467-78.

25. Herridge MS. Prognostication and intensive care unit outcome: the evolving role of scoring systems. Clin Chest Med 2003;24:751-62.

26. Afessa B, Gajic O, Keegan MT. Severity of illness and organ failure assessment in adult intensive care units. Crit Care Clin 2007;23:639-58.

27. Higgins TL. Quantifying risk and benchmarking performance in the adult intensive care unit. J Intensive Care Med 2007;22:141-56.

28. Minne L, Abu-Hanna A, de Jonge E. Evaluation of SOFA-based models for predicting mortality in the ICU: a systematic review. Crit Care 2008;12:R161.

29. Cholongitas E, Senzolo M, Patch D, Shaw S, Hui C, Burroughs AK. Review article: scoring systems for assessing prognosis in critically ill adult cirrhotics. Aliment Pharmacol Ther 2006;24:453-64.

30. Child CG, Turcotte JG. Surgery and portal hypertension. Major Probl Clin Surg 1964;1:1-85.

31. Pugh RN, Murray-Lyon IM, Dawson JL, Pietroni MC, Williams R. Transection of the oesophagus for bleeding oesophageal varices. Br J Surg 1973;60:646-9.

32. Kamath PS, Wiesner RH, Malinchoc M, Kremers W, Therneau TM Kosberg CL, et al. A model to predict survival in patients with endstage liver disease. Hepatology 2001;33:464-70.

33. Wiesner R, Edwards E, Freeman R, Harper A, Kim R, Kamath P, et al. Model for end-stage liver disease (MELD) and allocation of donor livers. Gastroenterology 2003;124:91-6.

34. Goldfarb G, Nouel O, Poynard T, Rueff B. Efficiency of respiratory assistance in cirrhotic patients with liver failure. Intensive Care Med $1983 ; 9: 271-3$
35. Moreau R, Hadengue A, Soupison T, Kirstetter P, Mamzer MF, Vanjak D, et al. Septic shock in patients with cirrhosis: hemodynamic and metabolic characteristics and intensive care unit outcome. Crit Care Med 1992;20:746-50.

36. Zauner CA, Apsner RC, Kranz A, Kramer L, Madl C, Schneider B, et al. Outcome prediction for patients with cirrhosis of the liver in a medical ICU: a comparison of the APACHE scores and liver-specific scoring systems. Intensive Care Med 1996;22:559-63.

37. Zauner C, Schneeweiss B, Schneider B, Madl C, Klos H, Kranz A, et al. Short-term prognosis in critically ill patients with liver cirrhosis: an evaluation of a new scoring system. Eur J Gastroenterol Hepatol 2000;12:517-22.

38. Wehler M, Kokoska J, Reulbach U, Hahn EG, Strauss R. Short-term prognosis in critically ill patients with cirrhosis assessed by prognostic scoring systems. Hepatology 2001;34:255-61.

39. Cholongitas E, Betrosian A, Senzolo M, Shaw S, Patch D, Manousou $\mathrm{P}$, et al. Prognostic models in cirrhotics admitted to intensive care units better predict outcome when assessed at $48 \mathrm{~h}$ after admission. $\mathrm{J}$ Gastroenterol Hepatol 2008;23:1223-7.

40. Teres D, Lemeshow S. Why severity models should be used with caution. Crit Care Clin 1994;10:93-110.

41. Chatzicostas C, Roussomoustakaki M, Notas G, Vlachonikolis IG, Samonakis D, Romanos J, et al. A comparison of Child-Pugh, APACHE II and APACHE III scoring systems in predicting hospital mortality of patients with liver cirrhosis. BMC Gastroenterol 2003;3:7

42. Afessa B, Kubilis PS. Upper gastrointestinal bleeding in patients with hepatic cirrhosis: clinical course and mortality prediction. Am J Gastroenterol 2000;95:484-9.

43. Brown JJ, Naylor MJ, Yagan N. Imaging of hepatic cirrhosis. Radiology 1997;202:1-16.

44. Yu II, Abola L. Predicting prognosis among cirrhotic patients: ChildPugh versus APACHE III versus MELD scoring systems. Phil J Gastroenterol 2006;2:19-24.

45. Ruttimann UE. Statistical approaches to development and validation of predictive instruments. Crit Care Clin 1994;10:19-35.

46. Lemeshow S, Hosmer DW Jr. A review of goodness of fit statistics for use in the development of logistic regression models. Am J Epidemiol 1982;115:92-106

47. Hanley JA, McNeil BJ. A method of comparing the areas under receiver operating characteristic curves derived from the same cases. Radiology 1983;148:839-43.

48. Youden WJ. Index for rating diagnosis tests. Cancer 1950;3:32-5.

49. Holt AW, Bury LK, Bersten AD, Skowronski GA, Vedig AE. Prospective evaluation of residents and nurses as severity score data collectors. Crit Care Med 1992;20:1688-91.

50. Domínguez L, Enríquez P, Alvarez P, de Frutos M, Sagredo V, López-Messa J, et al. Evaluation of the reproducibility of the data collection for the APACHE II, APACHE III adapted for Spain and the SAPS II in nine intensive care units in Spain. Med Intensiva 2008;32: $15-22$.

51. Janssens U, Graf C, Graf J, Radke PW, Königs B, Koch KC, et al. Evaluation of the SOFA score: a single-center experience of a medical intensive care unit in 303 consecutive patients with predominantly cardiovascular disorders. Sequential Organ Failure Assessment. Intensive Care Med 2000;26:1037-45.

52. Marshall JC, Cook DJ, Christou NV, Bernard GR, Sprung CL, Sibbald WJ. Multiple organ dysfunction score: a reliable descriptor of a complex clinical outcome. Crit Care Med 1995;23:1638-52.

53. Seneff MG, Zimmerman JE, Knaus WA. Outcome prediction for specfic conditions. In: Ayers SM, GrenvikA, Holbrook PR, Shoemaker WC, editors. Textbook of critical care. 3rd ed. Philadelphia: Saunders; 1995.p.1808-17

54. Luce JM, Wachter RM. The ethical appropriateness of using prognostic scoring systems in clinical management. Crit Care Clin 1994:10:229-41.

55. Seneff M, Knaus WA. Predicting patient outcome from intensive care: a guide to APACHE, MPM, SAPS, PRISM, and other prognostic scoring systems. J Intensive Care Med 1990;5:33-52. 